\title{
Coupling of detailed configuration kinetics and hydrodynamics in materials submitted to x-ray free-electron-laser irradiation
}

\author{
O. Peyrusse* \\ Univ. Bordeaux, CEA, CNRS, CELIA (Centre Lasers Intenses et Applications), UMR 5107, F-33400 Talence, France
}

(Received 3 July 2012; published 4 September 2012)

\begin{abstract}
We describe a model for the study of the interaction of short x-ray free-electron laser (XFEL) pulses with matter. Hydrodynamics is solved in one-dimensional planar geometry together with XFEL energy deposition, transport by thermal conduction and electron-ion energy exchange. Along with XFEL energy deposition, a proper detailed configuration accounting atomic physics model has been implemented in line with the calculations. Simulations typical of current XFEL conditions of irradiation are presented and discussed. Finally, the corresponding spectral emission of this XFEL-heated matter is calculated with the unresolved transition array formalism.
\end{abstract}

DOI: 10.1103/PhysRevE.86.036403

PACS number(s): 52.65.-y, 78.70.En, 42.55.Vc, 41.60.Cr

\section{INTRODUCTION}

The arrival of x-ray free-electron lasers (XFELs; FLASH, LCLS, SACLA, European XFEL [1-4]) delivering intense pulses of radiation up to $20 \mathrm{keV}$ of photon energy offers the opportunity to produce and probe matter in dense (solid) warm and/or hot states [5]. Indeed, these pulses (of duration less or of the order of $100 \mathrm{fs}$ ) are always much shorter than hydrodynamics time scales. So, after the X-ray pulse has interacted, the material can be in a dense (solid), highly ionized, yet crystalline (maybe exotic if numerous inner-shell holes are produced) state, of which many physical properties, such as equation of state (EOS), electronic structure, and phonon spectrum, are unknown. Also, how the material subsequently evolves into a more or less temperature-equilibrated expanding state is a major question because it is precisely a warm dense state, i.e., the subject of active investigations.

Noticeable studies focused on the characterization of matter after extreme ultraviolet (EUV) or X-FEL irradiation have already been reported [6-8]. Among the possibilities of producing exotic states with XFELs, one can mention the production of an "absorption-saturated" state in the soft x-ray regime [9] or the clear existence of a nonequilibrium electron distribution function [10].

Besides these specific studies, the production and the control of hot or warm dense matter by means of XFEL irradiation is of major interest in order to design well-defined experiments which aim at extracting specific information on this state of matter. The objective of this paper is to describe a simulation model for predicting the behavior and the evolution of bulk matter when it is submitted to a strong pulse of monochromatic $\mathrm{x}$-ray radiation.

One shall note early simulations of XFEL-heated matter based on standard hydrodynamics models designed for optical laser interaction with matter, in which x-ray absorption is based on the use of local thermodynamic equilibrium (LTE) opacity tables $[11,12]$. However, in the $\mathrm{x}$-ray regime, the largely dominant absorption process is photoionization, and, briefly speaking, the subsequent relevant atomic processes are Auger relaxation, electron impact ionization, electron-electron

*peyrusse@celia.u-bordeaux1.fr equilibration, three-body recombination, and, finally, electronion coupling (bulk matter heating) [13]. Note that some of these subsequent processes (Auger relaxation, collisional ionization, or recombination) take place only about 10-30 fs after the first photoionization processes occur. So, excepted for the energy transfer between the heated free electrons and the atoms, all of the previously quoted processes may occur during the interaction of the x-ray pulse itself. These remarks underline the need of a detailed treatment of the mentioned atomic processes in order to describe properly the time-dependent absorption mechanism. Going in this direction, Whittaker et al. [14] developed a static model describing the nonequilibrium absorption in carbon and iron samples. Their model, however, does not take into account collisional processes.

Here, we make use of a complete treatment of the necessary non-local thermodynamical equilibrium (NLTE) time-dependent atomic physics in the framework of a onedimensional hydrocode. Through simulation examples, our objective is to clarify the importance of some microscopic processes in order to get proper predictions of both temperatures, bulk matter evolution, and spectral emission.

In Sec. II, we give a detailed description of the model. Some particularly important computational details then are given in Sec. III. Finally, examples of simulations are discussed in Sec. IV.

\section{THEORETICAL MODEL}

The model can be conveniently partitioned into four aspects: (i) hydrodynamics, (ii) electron-ion coupling and electron thermal conduction, (iii) atomic physics and XFEL absorption, and (iv) equation of state. Many aspects or details of the method of solution are rather traditional in the general field of laser-matter interaction. The model is described in detail below in order to present a complete picture of the simulations reported on. In short terms, the material is assumed to be a charge-neutral mixture of electrons and ions. We then employ a one-fluid, one-dimensional, two-temperature model to account for thermal nonequilibrium and we solve rate equations of selected atomic configurations population densities for including both XFEL absorption, NLTE ionization dynamics, and spectral emission. 


\section{A. Hydrodynamics and the various gain and loss terms}

The basic variables are mass density, velocity, electron, and ion internal energy. The equations governing these quantities in planar geometry can be written as (in Lagrangian form)

$$
\begin{aligned}
\frac{d}{d t} \rho & =-\rho \frac{\partial v}{\partial x} \\
\frac{d}{d t} v & =-\frac{1}{\rho} \frac{\partial\left(P_{e}+P_{i}\right)}{\partial x} \\
\frac{d}{d t} \epsilon_{e} & =-\frac{P_{e}}{\rho} \frac{\partial v}{\partial x}+\frac{S_{e}}{\rho} \\
\frac{d}{d t} \epsilon_{i} & =-\frac{P_{i}}{\rho} \frac{\partial v}{\partial x}+\frac{S_{i}}{\rho}
\end{aligned}
$$

where $\rho$ is the mass density, $v$ is the fluid velocity, and $\epsilon_{e}$ and $\epsilon_{i}$ are the electron and ion internal energy (per mass unit), respectively. The two first equations are the equation for mass conservation and the equation of movement, respectively. The two remaining equations describe the evolution of internal energy (of a fluid cell) for electrons and ions, respectively. Hereafter, subscript $i$ is used for ion and $e$ for electron. $P_{e}, P_{i}$ are the electron and ion pressure, respectively. $S_{e}, S_{i}$ are two terms summarizing gain and/or loss of energy. Specifically, one has

$$
\mid \begin{aligned}
& S_{e}=-Q_{e i}-\frac{\partial q_{e}}{\partial x}+P_{a b s}\left(I_{\mathrm{XFEL}}\right) \\
& S_{i}=Q_{e i}
\end{aligned},
$$

where $Q_{e i}$ is energy transfer rate (per volume unit) of the electrons to the ions, the term $-\frac{\partial q_{e}}{\partial x}$ is the divergence of the electron thermal conduction flux $\left(q_{e}=-\kappa_{e} \nabla T_{e}\right.$, where $\kappa_{e}$ is the electron thermal conductivity), and $P_{\mathrm{abs}}\left(I_{\mathrm{XFEL}}\right)$ is the deposited power (per volume unit) by the (local) XFEL intensity $I_{\mathrm{XFEL}}$. These equations are numerically integrated, explicitly in time on a Lagrangian grid, except the third one, which gives rise to an equation for the electron temperature and, thus, requires a special treatment: a specific implicit twostep treatment, first taking into account the population kinetics and then followed by a standard treatment of the thermal conduction term. Important numerical details are given in Sec. III.

\section{B. Electron-ion coupling}

In a plasma, one usually writes the volumic energy transfer rate between the electrons and the ions as

$$
Q_{e i}=n_{e} \frac{3 m_{e}}{m_{i}} v_{e i} k\left(T_{e}-T_{i}\right)
$$

in which $v_{e i}$ is the usual (Spitzer) electron collision frequency $[15,16]$. In solids, one writes $Q_{e i}=g\left(T_{e}, T_{i}\right) \times\left(T_{e}-\right.$ $T_{i}$ ), where the factor $g$ describes the electron-phonon coupling. In that case, Eq. (3) allows one to define an effective collision frequency. In cold solids, this collision frequency no longer depends on $T_{e}$ but is governed by the scattering of electrons by phonons and, thus, depends on $T_{i}$. Factor $g$ has been evaluated for metals in the context of the free-electron gas model [17] and, more recently, by taking into account both the electron temperature (for externally heated valence electrons in solids) and a realistic density of state [18]. In a context where the matter goes from a solid state to a plasma through the so-called warm dense matter state, one needs a more general formulation. Following Eidmann et al. [19],

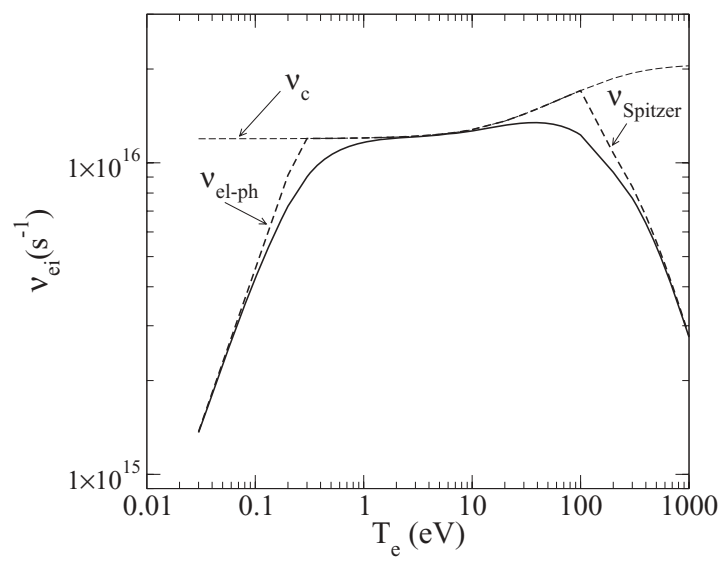

FIG. 1. Collision frequency of solid $\mathrm{Al}$ as a function of temperature. The solid line is the smoother interpolation (see text). The dashed line is the formulation given in Ref. [19]. The upper (limit) curve is the cutoff frequency (see text).

we will approximate the effective collision frequency by interpolating between the electron-phonon collision frequency at low temperature and the Spitzer formula at high temperature. These authors also introduced a cutoff frequency $v_{c}$ required by the constraint of having an electron mean free path always greater than the ion sphere radius. For the electron-phonon collision frequency, they use the parameter-dependent expression $v_{\mathrm{el}-\mathrm{ph}}=2 k_{s} \frac{e^{2} k T_{i}}{\hbar v_{F}}$, where the parameter $k_{s}$ must be adjusted to recover a correct (known) electron coupling coefficient $g$ [through Eq. (3)] for the material of interest (typically $k_{s}=13$ for aluminum). Then, as they use an harmonic mean limited by the cutoff frequency, i.e., $v_{e i}=\min \left(v, v_{c}\right)$ with $v^{-1}=v_{\text {Spitzer }}^{-1}+v_{\text {el-ph }}^{-1}$, it is probably more adequate to use a smoother interpolation like $v_{e i}^{-2}=v_{\text {Spitzer }}^{-2}+v_{\text {el-ph }}^{-2}+v_{c}^{-2}$ [20]. This rather artificial electron collision frequency underlines the lack of a usable formulation in the warm dense regime but faute de mieux we shall accept this. The behavior of this electron collision frequency for solid aluminum $(\rho=2.7$ $\left.\mathrm{cm}^{-3}\right)$ and equal temperatures $\left(T_{e}=T_{i}\right)$ is displayed in Fig. 1. Average ionization has been assumed to be at LTE. The dashed curve corresponds to the original formulation of Eidmann et al. while the plain one is the smoother interpolation. Also shown is the cutoff frequency given by the requirement $\lambda_{e}>r_{o}$, where $\lambda_{e}$ is the electron mean free path and $r_{o}$ is the ion-sphere radius.

One shall note that the electron collision frequency is also important for calculating the electron thermal conductivity, $\kappa_{e}$. In the plasma regime, the well-known Spitzer's formulation [15] which depends on the electron collision frequency, is available. This formulation can be extended to low temperatures by using for $v_{e i}$ the same interpolation as discussed previously. However, to get consistency with the cold thermal conductivity (Wiedemann-Franz's law and its correct coefficient), it is necessary to use a different parameter, $k_{s}$ (typically, $k_{s}=2.3$ for $\mathrm{Al}[19]$ ).

\section{NLTE atomic physics and XFEL absorption \\ 1. Excitation and ionization dynamics}

Along with the above equations, one follows a set of (nonrelativistic) atomic configuration population densities 
$\left\{N_{c}\right\}$ by solving (in each Lagrangian cell) a system of timedependent rate equations,

$$
\frac{d N_{c}}{d t}=-\sum_{c^{\prime}} N_{c} R_{c c^{\prime}}+\sum_{c^{\prime}} N_{c^{\prime}} R_{c^{\prime} c}
$$

where $R_{c c^{\prime}}$ denotes a total rate coefficient for population transfer from configuration $c$ to configuration $c^{\prime}$. This approach, which is the basis for most NLTE calculations, is the so-called collisional-radiative model. It describes each atomic system in terms of an affordable number of atomic levels. The interested reader will find a discussion about complexity and difficulties associated with this task in Refs. [21,22].

The atomic level structure employed here is configuration averaged in the sense that only atomic configurations are considered and that all rates corresponding to collisional and radiative processes are properly configuration averaged [23] with quantum-mechanical formulations. The rate coefficients that are used to calculate the populating or depopulating rates $R_{c c^{\prime}}$ are obtained using various techniques. The processes included and the basic methods used for calculating the corresponding rate coefficients are summarized below.

1. Collisional excitation: Distorted-wave approximation including exchange.

2. Spontaneous radiative decay: Dipolar-electric approximation [24].

3. Collisional ionization: We used here a prescription (given in Refs. [25-27]) based on a fit of Coulomb-Bornexchange calculations.

4. Autoionization: Standard formulation of an autoionization rate based on the evaluation of matrix elements of the electrostatic repulsion operator that connects an (abovethreshold excited) $N+1$ bound electron state and a state built from $N$ bound orbitals and one continuum orbital (for a recent review, see, for instance, Ref. [28]).

5. Photoionization: Dipolar-electric approximation for the cross section [24].

Also, collisional deexcitation, collisional recombination, electronic capture, and radiative recombination rates are all calculated as the detailed balance of the corresponding opposite rates listed above: (1), (3), (4), and (5), respectively. Rates associated with the coupling of the XFEL are detailed below.

For convenience, we use the following notation for a configuration: $c \equiv \ell_{\alpha}^{n_{\alpha}} \ell_{\beta}^{n_{\beta}} \ell_{\gamma}^{n_{\gamma}} \ldots$, where $\ell_{\alpha}^{n_{\alpha}}$ stands for orbital $\alpha$ of orbital momentum $\ell_{\alpha}$ and of occupation $n_{\alpha}$. So, between two specified configurations $c \equiv \ell_{\alpha}^{n_{\alpha}} \ell_{\beta}^{n_{\beta}} \ell_{\gamma}^{n_{\gamma}} \ldots$ and $c^{\prime} \equiv$ $\ell_{\alpha}^{n_{\alpha}-1} \ell_{\beta}^{n_{\beta}} \ell_{\gamma}^{n_{\gamma}} \ldots$ and, given a local XFEL intensity $I_{\mathrm{XFEL}}(x)$ (in $\mathrm{W} / \mathrm{cm}^{2}$ ) of photon energy $h v_{o}>\chi_{c^{\prime} c}$ (where $\chi_{c^{\prime} c}$ is the energy difference between $c$ and $c^{\prime}$ ), the photoionization rate connecting two configurations $c$ and $c^{\prime}$ is

$$
W_{c c^{\prime}}\left(s^{-1}\right)=6.241 \times 10^{18} I_{\mathrm{XFEL}}(x) \frac{\sigma_{c c^{\prime}}\left(h v_{o}\right)}{h v_{o}},
$$

where $\sigma_{c c^{\prime}}\left(h v_{o}\right)$ is photoionization cross section taken at $h v_{o}$. In Eq. (5), $h v_{o}$ is in eV. From the principle of detailed balance, one easily gets the inverse rate, namely the stimulated recombination rate

$$
W_{c^{\prime} c}=1.656 \times 10^{-22} \times n_{e} \frac{g_{c}}{g_{c^{\prime}}} \frac{\exp \left[\left(\chi_{c^{\prime} c}-h v_{o}\right) / k T_{e}\right]}{\left(k T_{e}\right)^{3 / 2}} W_{c c^{\prime}} .
$$

Between two configurations $c \equiv \ell_{\alpha}^{n_{\alpha}} \ell_{\beta}^{n_{\beta}} \ell_{\gamma}^{n_{\gamma}} \ldots$ and $c^{\prime} \equiv$ $\ell_{\alpha}^{n_{\alpha}-1} \ell_{\beta}^{n_{\beta}+1} \ell_{\gamma}^{n_{\gamma}} \ldots$, the photoexcitation rate corresponding to the jump $\alpha \rightarrow \beta$ reads,

$$
Q_{c c^{\prime}}=1.578 \times 10^{-5} I_{\mathrm{XFEL}}(x) \frac{g_{c^{\prime}}}{g_{c}} A_{c^{\prime} c} \frac{\tilde{\phi}_{c^{\prime} c}\left(h v_{o}\right)}{\left(h \tilde{v}_{c^{\prime} c}\right)^{3}},
$$

where $A_{c^{\prime} c}$ is the configuration-average radiative transition rate between $c^{\prime}$ and $c$ [23]. $h \tilde{\nu}_{c^{\prime} c}$ and $\tilde{\phi}_{c^{\prime} c}$ are, respectively, the position and the statistical (Gaussian) profile associated with the set of radiative transitions (fine-structure lines) between $c^{\prime}$ and $c$. These quantities can be properly calculated from the unresolved transition array (UTA) theory [29]. The inverse rate, namely the stimulated emission rate is easily obtained from the relation $g_{c} Q_{c c^{\prime}}=g_{c^{\prime}} Q_{c^{\prime} c}$.

Finally, once the rate equation has been solved, the local average charge is written as $\langle Z\rangle=\left(M_{i} / \rho\right) \sum_{c} z_{c} N_{c}$, where $M_{i}$ is the ion mass and $z_{c}$ is the charge of configuration $c$. The electron density then is such that $n_{e}=\langle Z\rangle\left(\rho / M_{i}\right)$.

\section{Absorption of XFEL radiation}

If one neglects the free-free absorption (inverse Bremsstrahlung), the power deposited (per volume unit) by the XFEL in Eq. (2) is just

$$
P_{\mathrm{abs}}\left(I_{\mathrm{XFEL}}\right)=\sum_{c, c^{\prime}} N_{c} W_{c c^{\prime}}+\sum_{c, c^{\prime}} N_{c} Q_{c c^{\prime}} .
$$

In turn, this local deposited power can be written (in CGS unit) as $P_{\text {abs }}=k_{v_{o}}\left(I_{\mathrm{XFEL}} \times 10^{7}\right)$, which defines the local opacity $k_{v_{o}}$ $\left(\mathrm{cm}^{-1}\right)$ at the XFEL frequency.

Taking a few tenths of a micron as a typical EUV/x-ray attenuation length, one can neglect the retardation times in the $\mathrm{x}$-ray transport. Therefore, the local XFEL intensity in the material is computed by solving the radiation transport along a $h v_{o}$ photon path with a simple cell to cell exponential attenuation.

\section{Choice of atomic configurations}

In our approach, a simulation starts from a precalculation of all of the needed rates or cross sections for a preselected well-balanced set of configurations. Unlike the purely thermal optically thin plasmas where excitation or ionization is mainly driven by electron collisional processes, XFEL absorption may result from inner-shell processes and the preselected configuration set must describe the possible photoionization processes from stable and/or autoionizing configurations. In addition, it is important to include valence-shell singly excited configurations allowing for collisional ionization and three-body recombination to occur. To limit the size of the rate-equation system [Eq. (4)] and, depending on the XFEL photon energy $h v_{o}$, we do not include inner-shell ionized configurations which cannot be accessed through single-photon ionization. Also, depending on a guessed maximum ionization, we do not necessarily include all the ionization stages. In that sense, our selected set is case dependent. Following the sequential chain of one-photon photoionization processes, one can illustrate, for carbon, the choice of the essential configurations in the case where $K$-shell photoionization is allowed. Starting from the ground configuration in C-I, i.e., 
$1 s^{2} 2 s^{2} 2 p^{2}$, photoionization gives for C-II,

$$
\begin{aligned}
& 1 s^{2} 2 s^{2} 2 p \\
& 1 s^{2} 2 s 2 p^{2} \\
& 1 s^{2} 2 s^{2} 2 p^{2}
\end{aligned}
$$

and, for C-III,

$$
\begin{gathered}
1 s^{2} 2 s^{2} \\
1 s^{2} 2 s 2 p \\
1 s 2 s^{2} 2 p \\
1 s^{2} 2 p^{2} \\
1 s 2 s 2 p^{2} \\
2 s^{2} 2 p^{2},
\end{gathered}
$$

and so on for CIV, CV, and CVI, where hollow configurations may occur. Note that this set remains partial since the more traditional valence-shell singly excited configurations must be added in each ion species.

\section{Density effects}

It is well known that density effects on bound electron energy levels lead to the phenomenon of continuum lowering (CL). Moreover, most of the population kinetics calculations indicate that the main impact of the density is continuum lowering and that additional corrections beyond this phenomenon are uncertain [30]. Therefore, most of the collisional-radiative models take into account density effects in an approximate way via a lowering of the ionization potentials. So, for a particular configuration $c$, the effective ionization potential reads $\chi_{c}^{\prime}=\chi_{c}+\Delta I_{c}$, where $\Delta I_{c}$ is the continuum lowering (i.e., a negative quantity to be applied to lower the isolated-atom value of $\chi_{c}$ ). Note that recent experiments on solid-density plasmas produced by XFEL irradiation have shown that this problem could be particularly acute [31]. A simple theory of CL is that of Stewart and Pyatt [32]. Their result is summarized by the formula [33]

$$
\Delta I_{c}=-\frac{3}{2} \frac{Z_{c} e^{2}}{R_{c}}\left\{\left[1+\left(\frac{D}{R_{c}}\right)^{3}\right]^{2 / 3}-\left(\frac{D}{R_{c}}\right)^{2}\right\},
$$

which is the CL for a configuration of charge $Z_{c}$ immersed into a plasma having Debye length $D$ and ion-sphere radius $R_{c}$ [here defined such that $(4 \pi / 3) R_{c}^{3} n_{e}=Z_{c}$ ]. In the present work, we have chosen to use the same value $\Delta I$ for all configurations by taking $Z_{c} \simeq\langle Z\rangle$ in the previous equation (note that this gives also a unique value of $R_{c}$ ). Furthermore, in the simulations, we fix the initial value of $\langle Z\rangle$ at some value corresponding to the known valence ionization (e.g., $\langle Z\rangle=3$ for solid-state cold $\mathrm{Al},\langle Z\rangle=1$ for solid-state cold $\mathrm{Ag}$, etc.). Therefore, the photoionization thresholds must be consequently shifted of the corresponding CL. In principle, the application of a CL correction brings a significant reduction in the number of bound configurations in the sense that configurations with $E_{c}>\chi_{c}^{\prime}$ must be eliminated from the calculation. In order to keep a constant dimension for the rate-equation systems throughout a whole simulation, we chose to keep all the configurations. However, in an ion species where all configurations satisfy the previous condition of nonexistence, we impose an artificially large ionization rate along with a zero recombination rate.

Finally, to get a smoother behavior of the ionization in some cases, we found convenient to use Zimmerman-More's prescription consisting in reducing the statistical weights by a density-dependent reduction factor [34].

\section{Equation of state}

Consistent with the two-temperature model presented above, and despite the fact that we are in a NLTE regime, we need a formulation for both $\left(\epsilon_{i}, P_{i}\right)$ and $\left(\epsilon_{e}, P_{e}\right)$.

The ion EOS used is the simple QEOS one [35], based on an unpublished work of Cowan. In short, for all elements, the formulation starts from a fit (function of $Z$ and $\rho$ ) of the three quantities, the melting temperature $T_{m}$, the Debye temperature $\theta_{D}$, and the Gruneisen coefficient $\gamma_{s}$, respectively. Then, given $\rho, T_{i}$, and two scaling variables $u=\theta_{D} / T_{i}, w=T_{m} / T_{i}$, the model gives $\left(\epsilon_{i}, P_{i}\right)$ for the three regimes (the fluid phase, the high-temperature solid, and the low-temperature solid).

The electron internal energy $\epsilon_{e}$ is expressed as a sum of a zero temperature, or cold $\left(T_{e}=0\right)$, part with a purely thermal $\left(T_{e} \neq 0\right)$ part,

$$
\rho \epsilon_{e}=E_{\text {free }}\left(\rho, T_{e}\right)+\sum_{c}\left(E_{c}-E_{c_{1}}\right) N_{c}\left(\rho, T_{e}\right)+E_{\text {cold }}(\rho),
$$

where the first term $\left(E_{\text {free }}\right)$ is the thermal contribution of free electrons while the second term, a sum over all excitation energies, is the contribution of bound electrons [note that $E_{c}-$ $E_{c_{1}}$ is the energy of configuration $c$ measured with respect to the ground (and neutral) configuration energy $E_{c_{1}}$ ]. In the present work, the cold contribution $E_{\text {cold }}$ is taken (together with $P_{\text {cold }}$ ) from the scaled binding-energy model [36]. Exact expression of $E_{\text {free }}$ is that of the quantum electron gas, namely $E_{\text {free }}=a_{1}\left(k T_{e}\right)^{5 / 2} F_{3 / 2}\left(\mu / k T_{e}\right)-\frac{3}{5} N_{e} k T_{e}$ in which $F_{3 / 2}$ is a well-known Fermi integral and $a_{1}=4 \pi\left(2 m_{e}\right)^{3 / 2} / h^{3}$. Considering the low-temperature (degenerate) limit of $E_{\text {free }}$, i.e., $E_{l}=N_{e} \epsilon_{F} \frac{\pi^{2}}{4}\left(\frac{k T_{e}}{\epsilon_{F}}\right)^{2}$, and the high-temperature (perfect gas) limit $E_{h}=\frac{3}{2} N_{e} k T_{e}$, we found convenient (in particular for computing derivatives with respect to $T_{e}$ ) to use the following interpolation:

$$
\frac{1}{\left(E_{\text {free }}\right)^{\alpha}}=\frac{1}{\left(E_{l}\right)^{\alpha}}+\frac{1}{\left(E_{h}\right)^{\alpha}}
$$

with $\alpha=1.45$. A comparison of this interpolation with the exact expression for solid $\mathrm{Al}$ is shown in Fig. 2, along with the two limits. Also shown is the QEOS contribution of ions.

Finally, the electron pressure is needed and is formulated as follows: $P_{e}=P_{\text {free }}\left(\rho, T_{e}\right)+P_{\text {cold }}(\rho)$ with $P_{\text {free }}$ given by the relation $3 P_{\text {free }}=2 E_{\text {free }}$.

It is important to keep in mind that this formulation of the electron EOS assumes an instantaneous thermalization of the electrons which implies that photoelectrons and Auger electrons resulting from the XFEL energy deposition thermalize very quickly. For XUV photons and low fluence, it has been shown that this is not true [10]. In our global context, future investigations will show if this approximation is not a too severe one. 


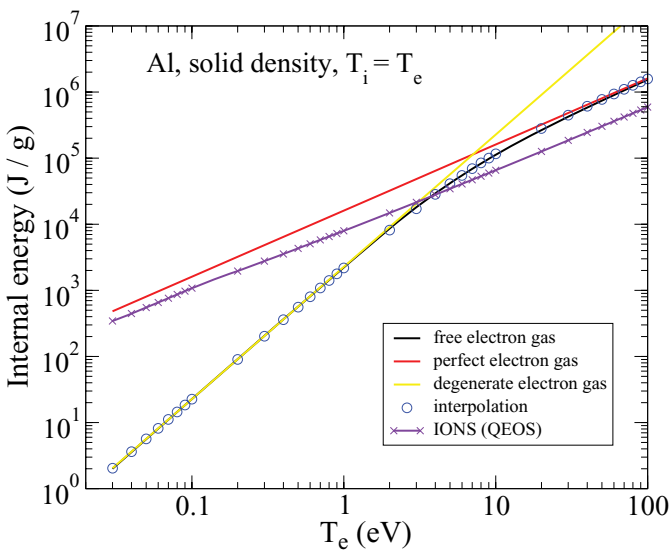

FIG. 2. (Color online) Internal energy of free electrons in $\mathrm{Al}$ as a function of the temperature at $\rho=2.7 \mathrm{~cm}^{-3}$. Also shown is the internal energy of ions according to the QEOS model.

\section{COMPUTATIONAL DETAILS}

We have developed a computer program to solve the equations presented above in planar geometry and thereby to handle arbitrary x-ray pulses, although we limit our study to Gaussian pulses in this paper.

A each time step, we track the zone boundaries (velocities and positions). This gives a new density in each Lagrangian cell. We then reactualize the internal energies (ionic and electronic) in three steps. In the first step, we take into account explicitly both the hydrodynamic contribution $\left(-\frac{P}{\rho} \frac{\partial v}{\partial x}\right)$, the equilibration term $\left(T_{e}, T_{i}\right)$, and the XFEL energy deposition (for $\epsilon_{e}$ ). In the second step, we treat specifically the equation for the (partially updated in the first step) electron internal energy $\epsilon_{e}$ and the atomic physics (i.e., the rate-equation system). This step is addresssed in more detail below. Last, in the third step, the electron thermal conduction term is calculated implicitly. This is done in one iteration, i.e., we neglected the variation of the temperature dependence of the thermal conductivity over a finite-difference time step.

In situations where the ionization varies significantly over the hydrodynamics time scale (due to a fast XFEL intensity variation or a fast temperature variation), we may have large numerical oscillations in the electron energy balance. In other words, the ionization energy term [the second term in the right of Eq. (10)] may cause a numerical instability due to the rapid variation of the population kinetics. This difficulty is controlled either by working explicitly (i.e., without taking a specific care of the coupling between the electron energy balance and the rate equation system) and by imposing a very small time step (of the order of $10^{-17} \mathrm{~s}$ or less during the $\mathrm{x}$-ray pulse) or, more efficiently, in the following manner: Suppose we write the equation for the electron internal energy [third equation in system (1)] as

$$
\frac{d}{d t} \epsilon_{e}=W
$$

where $W$ contains all sources, losses, or hydrodynamics terms. This equation must be solved together with the rate equation system,

$$
\frac{d \mathbf{N}}{d t}=\mathbf{T}\left(T_{e}\right) \cdot \mathbf{N}
$$

where a rate matrix $\mathbf{T}$ has been introduced along with the vector of configuration populations $\mathbf{N}$. Introducing a vector of atomic configuration energies $\mathbf{E}$ and using Eq. (10), the form given to $\epsilon_{e}$ can shortened as

$$
\epsilon_{e}=f\left(T_{e}\right)+\mathbf{E} \cdot \mathbf{N}
$$

Let us suppose the solution to be known at instant $t$ (superscript "-"). We wish to know the solution at $t+\Delta t$ (superscript "+"). For a given $W$, the updated value of $\epsilon_{e}$ is just $\epsilon_{e}^{+}=\epsilon_{e}^{-}+W \Delta t$ so our problem is now to get $\left(\mathbf{N}, T_{e}\right)$ from Eqs. (13) and (14). A fully implicit discretization of these equations gives the system

$$
\begin{aligned}
{\left[\mathbf{1}-\Delta t \mathbf{T}\left(T_{e}^{+}\right)\right] \cdot \mathbf{N}^{+} } & =\mathbf{N}^{-} \\
f\left(T_{e}^{+}\right)+\mathbf{E} \cdot \mathbf{N}^{+} & =\epsilon_{e}^{+}
\end{aligned}
$$

This system can be reformulated by defining a function $F$ acting on the set $\left(\mathbf{N}^{+}, T_{e}^{+}\right) . F$ is defined as

$$
F_{i}=\sum_{j=1}^{N} U_{i j} N_{j}^{+}-N_{i}^{-}
$$

for $i=1, N$ ( $N$ is the total number of included atomic configurations) and for $i=N+1$,

$$
F_{N+1}=\sum_{j=1}^{N} E_{j} N_{j}^{+}+f\left(T_{e}^{+}\right)-\epsilon^{+} .
$$

In Eq. (16), matrix elements of $\mathbf{U}$ are defined as $U_{i j}=$ $\delta_{i j}-\Delta t T_{i j}\left(T_{e}^{+}\right)$. So, system (15) reduces to $N+1$ functional relations to be zeroed, i.e.,

$$
F_{i}\left(N_{1}^{+}, N_{2}^{+}, \ldots, N_{N}^{+}, T_{e}^{+}\right)=0, \quad i=1, N+1 .
$$

To solve these equations, we linearize the entire system and solve for corrections to the current solution. The $\left(\delta \mathbf{N}, \delta T_{e}\right)$ found in this manner are added to the current values of $\left(\mathbf{N}, T_{e}\right)$ (which are the $\left(\mathbf{N}^{-}, T_{e}^{-}\right)$when starting the procedure) and the process continues until the changes are negligible. More precisely, with the superscript $q$ standing for iteration $q$, the linearization gives,

$$
\begin{aligned}
& F_{i}\left(\mathbf{N}^{(q)}+\delta \mathbf{N}, T_{e}^{(q)}+\delta T_{e}\right) \\
& \quad \simeq F_{i}\left(\mathbf{N}^{(q)}, T_{e}^{(q)}\right)+\sum_{j=1}^{N} \frac{\partial F_{i}}{\partial N_{j}} \delta N_{j}+\frac{\partial F_{i}}{\partial T_{e}} \delta T_{e} .
\end{aligned}
$$

Zeroing the new $F_{i}$ gives the linear system $\mathbf{J}^{(q)} \cdot \delta \mathbf{x}=$ $-\mathbf{F}\left(\mathbf{x}^{(q)}\right)$ with $\mathbf{x}^{(q)} \equiv\left(\mathbf{N}^{(q)}, T_{e}^{(q)}\right)$ and, $J_{i j}=\frac{\partial F_{i}}{\partial N_{j}} \quad(j=1, N)$, $J_{i N+1}=\frac{\partial F_{i}}{\partial T_{e}}$. Note that this scheme is equivalent to NewtonRaphson iterations. We found it efficient to include the constraint $\sum_{j} N_{j}=N_{\text {ion }}$ by replacing one of the Eqs. (16) by the equation $F_{i}=\sum_{j} N_{j}^{+}-N_{\text {ion }}$. Also, for each step, one can check that the sum $\sum_{j} \delta N_{j}$ is essentially zero. It should be noted that both the rates and $f\left(T_{e}\right)$ depend also on the electron density in Eqs. (15). Because the dependence on the electron density is much less stiff than the temperature dependence, this 
situation can be overcome by a simple iteration on the electron density.

Finally, we introduced an artificial pseudoviscosity pressure $w$ [37] to prevent spurious oscillations in the compressed zones. $w$ was taken to be [38]

$$
w=c_{1} \rho c_{s}|\Delta v|+c_{2} \rho(\Delta v)^{2},
$$

where $\Delta v$ is the velocity difference across a zone. $c_{1}$ and $c_{2}$ are constants of order unity and $c_{s}$ is the sound speed. Also, Eq. (19) is applied only when a zone is being compressed.

\section{SIMULATIONS AND DISCUSSION}

In this section, we will discuss in detail the absorption and the hydrodynamics behavior of a few selected cases, namely the interaction of a XUV pulse typical of the FLASH facility (92-eV photons at $10^{16} \mathrm{~W} / \mathrm{cm}^{2}$ or below) and the interaction of much more energetic photon pulses at higher intensities typical of the LCLS facility. The target material is aluminum initially at room temperature $\left(T_{e}=T_{i}=0.03 \mathrm{eV}\right)$ and solid density $\left(\rho=2.7 \mathrm{~g} / \mathrm{cm}^{3}\right)$. We set the heat flux inhibition parameter to the free streaming limit $f=0.6$. Simulations performed with a lower value $f=0.15$ did not show any difference.

\section{A. EUV (92-eV) irradiation of a thick target}

We present and discuss here the simulation corresponding to the interaction of a short [20 fs full width at half maximum (FWHM)], intense $\left(10^{16} \mathrm{~W} / \mathrm{cm}^{2}\right)$ pulse of $92-\mathrm{eV}$ photons with a thick $(1.2 \mu \mathrm{m})$ aluminum target. The word "thick" is relative to the full opacity (zero transmission) of such a foil to $92-\mathrm{eV}$ photons. Our atomic model in that case includes 405 atomic configurations.

Figures 3-5 shows snapshots of the spatial profiles of $\rho, T_{e}$, $T_{i}$, respectively, from the time of maximum XFEL intensity ( $t=27 \mathrm{fs}$ ) up to $10 \mathrm{ps}$. The XFEL radiation is coming from the right. In Fig. 4, one notes an electron temperature profile at the time of maximum XFEL intensity indicating a penetration depth of about $0.5 \mu \mathrm{m}$, i.e., quite deep into the solid so atomic kinetics takes place at solid density. One notes that the maximum electron temperature is reached a bit after the

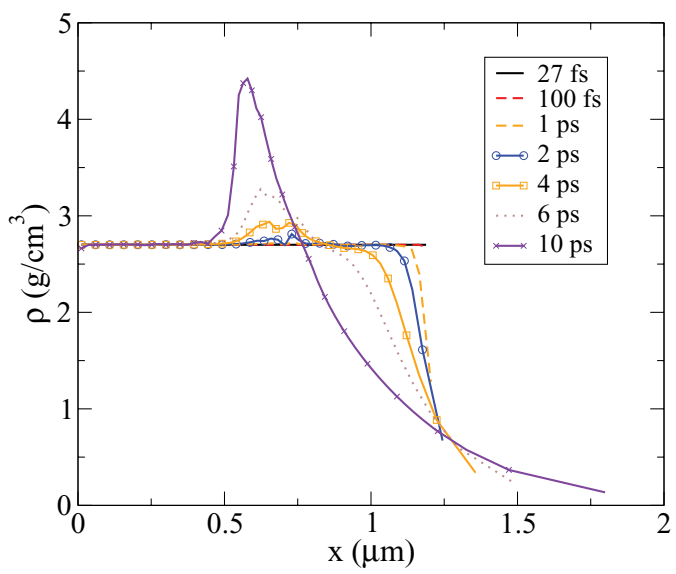

FIG. 3. (Color online) Snapshots of density profile for various times. The XFEL $\left[10^{16} \mathrm{~W} / \mathrm{cm}^{2}\right.$ intensity, 20 -fs full width at half maximum (FWHM), 92-eV photon energy] comes from the right.

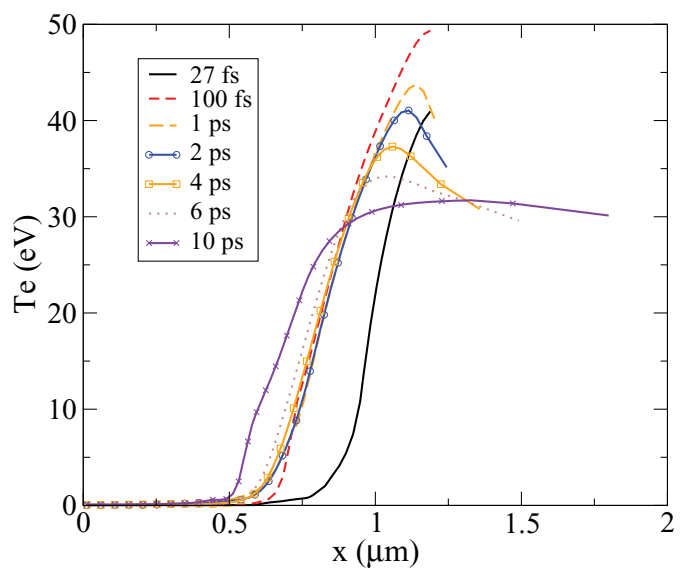

FIG. 4. (Color online) Snapshots of electron temperature profile for various times. XFEL conditions as in Fig. 3.

pulse has switched off (see the curve at $t=100 \mathrm{fs}$ ). Then, a heat wave propagates into the solid, $T_{i}$ tends to equilibrate with $T_{e}$ (see Fig. 5), the heated material expands (as seen in Fig. 3), and, later, after a few picoseconds, a shock wave develops in the opposite direction. A more global picture of what happens is plotted in Fig. 6, which displays the evolution of various quantities summed over all the cells (and weighted by the corresponding surfacic masses). (The thick solid curve corresponds to the total power deposited by the XFEL, the thin line is the electron internal energy, the dashed line is the total ion internal energy, the dotted line is the matter kinetic energy, and the long dashed line is the total radiated power). One can follow how the XFEL photons deposit their energies on the electrons, which in turn and after some delay transfer energy to the ions. Then, as seen on the kinetic energy curve, the matter starts to move. The time history of the power radiated by the resulting plasma (bound-bound and free-bound transitions) is also given. Starting significantly after the pulse has switched on, plasma radiation decreases a few ps after. It is instructive to look at the corresponding radiation spectra as shown in Fig. 7. These spectra, calculated using the UTA formalism, correspond to the front side radiation (along a line of sight parallel to the XFEL direction). More precisely, the radiative

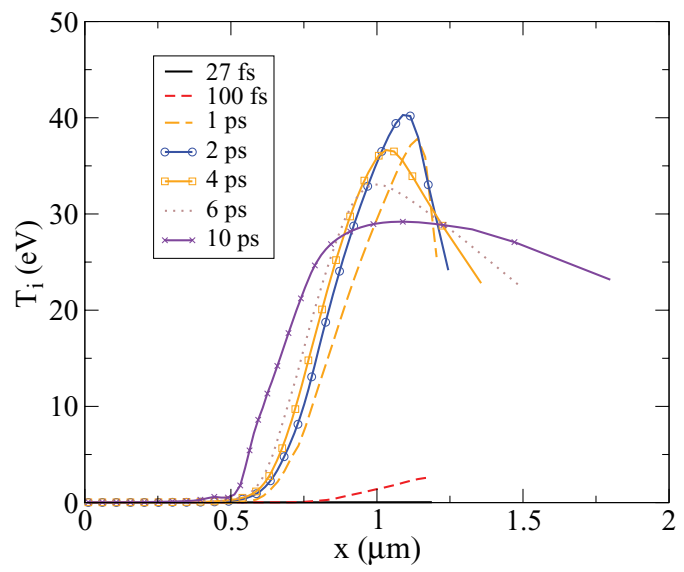

FIG. 5. (Color online) Snapshots of ion temperature profile for various times. XFEL conditions as in Fig. 3. 


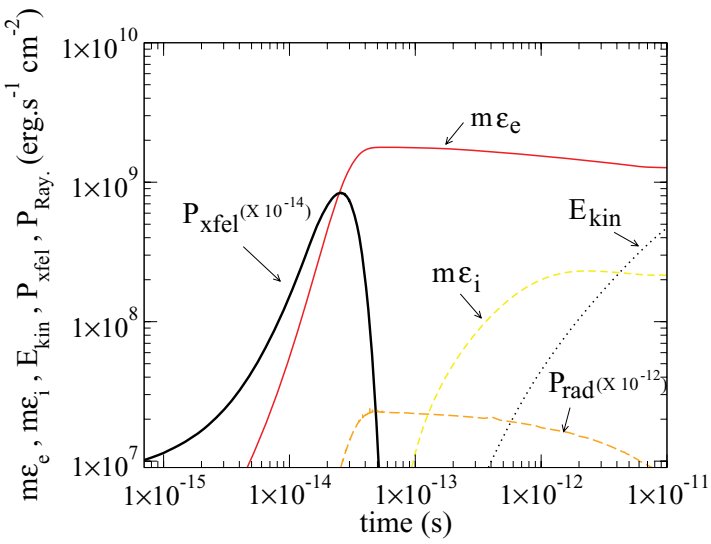

FIG. 6. (Color online) Total power deposited by the XFEL $\left(\mathrm{erg} / \mathrm{cm}^{2} / \mathrm{s}\right)$, total electron internal energy $\left(\mathrm{erg} / \mathrm{cm}^{2}\right)$, total ion internal energy $\left(\mathrm{erg} / \mathrm{cm}^{2}\right)$, total matter kinetic energy $\left(\mathrm{erg} / \mathrm{cm}^{2}\right)$, and total radiated power $P_{\text {rad }}\left(\mathrm{erg} / \mathrm{cm}^{2} / \mathrm{s}\right)$. XFEL conditions as in Fig. 3 . To display all quantities in the same figure, a multiplier has been applied to $P_{\text {xfel }}$ and $P_{\text {rad }}$.

transfer equation has been solved from cell to cell to built an emerging spectral intensity. The spectra displayed in Fig. 7 correspond to some of the snapshots of Figs. 3-5. It is worth noting that some spectral features carry information on ionic emitters existing only at some specific instants. This seems to be particularly the case for the early instants.

One shall note that present simulations were performed using the previously discussed (Sec. II B) standard set of parameters for the electron collision frequency as used in the electron-ion equilibration term and the thermal conduction coefficient, respectively. To show the effect of a particular choice of one of these parameters, we display in Fig. 8 a comparison of the ion temperature profiles at three different times, from two calculations using two different choices of the paramater $k_{s}$ used in the electron-ion equilibration frequency. Solid lines correspond to the "standard" choice for aluminum, $k_{s}=13.0$ [19] while dashed lines correspond to the choice $k_{s}=1.0$ (the most direct choice without any information concerning the electron-ion coupling factor in a cold material). Comparing these curves at $t=27 \mathrm{fs}$ and $t=$ $100 \mathrm{fs}$, respectively, shows how the choice of this parameter

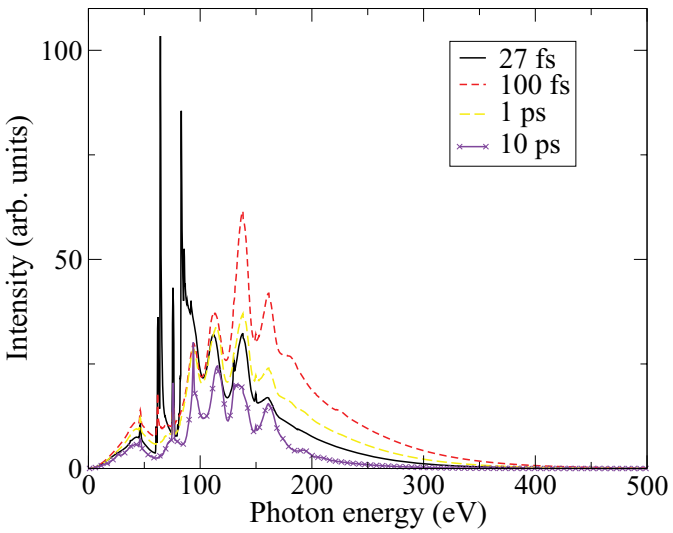

FIG. 7. (Color online) XUV spectra of the radiated energy at some instants. XFEL conditions as in Fig. 3.

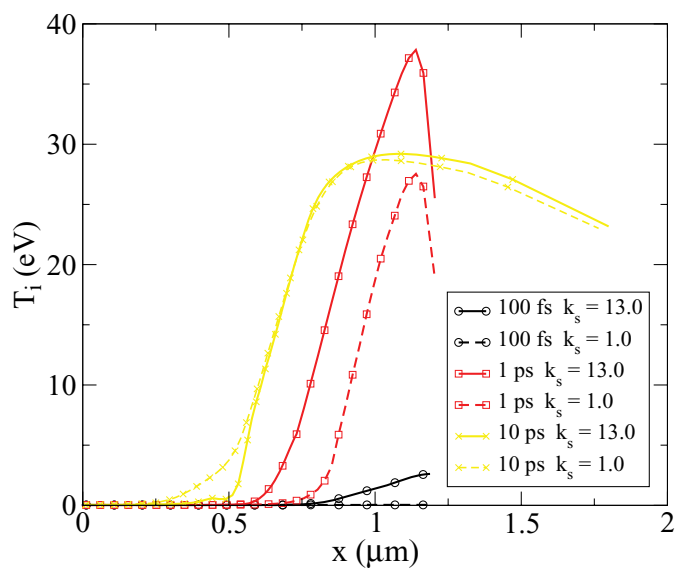

FIG. 8. (Color online) Effect of a change of the free parameter $k_{s}$ involved in the electron-ion relaxation (see text) on the ion temperature profiles. XFEL conditions as in Fig. 3.

strongly modifies the equilibration time. Despite the very crude choice $k_{s}=1.0$, the somewhat artificial definition of the interpolation formula (discussed in Sec. II B) underlines the lack of a usable and accurate formulation of the electron-ion relaxation times in warm dense matter.

Finally, we would like to stress the influence of some collisional effects on the heating itself. Here we artificially cut the three-body recombination by multiplying the corresponding rates by a reduction factor in the rate equation systems. The effect is shown in Fig. 9 on the electron temperature profile for a time close to the heating maximum. The upper curve is the standard calculation, i.e., without modifying the three-body recombination rates. One clearly sees that the heating is strongly affected. The reason is that, during the XFEL pulse, collisional recombination supplies a population flux of $1 s^{2} 2 s^{2} 2 p^{6}$ configurations from which XFEL absorption can again take place.

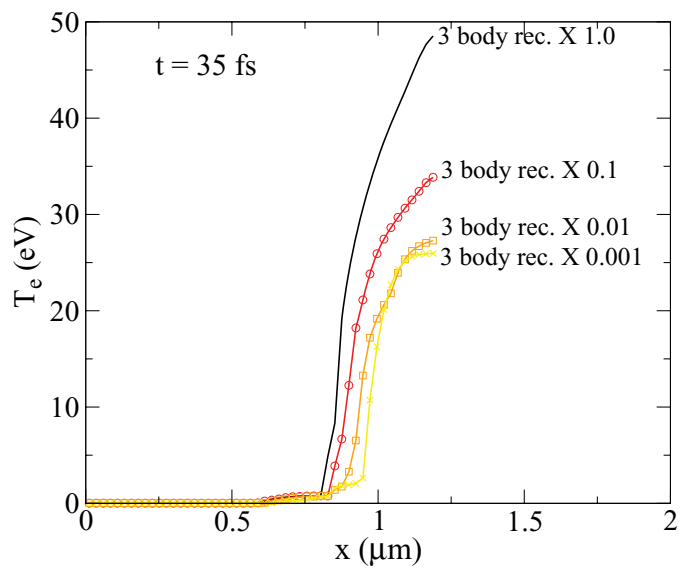

FIG. 9. (Color online) Influence of the three-body recombination process on the heating. Calculated profiles of $T_{e}$ for a time close to the maximum of heating. The profiles correspond to four different calculations where all the three-body recombination rates have been multiplied by the reduction factors reported in the figure. XFEL conditions as in Fig. 3. 


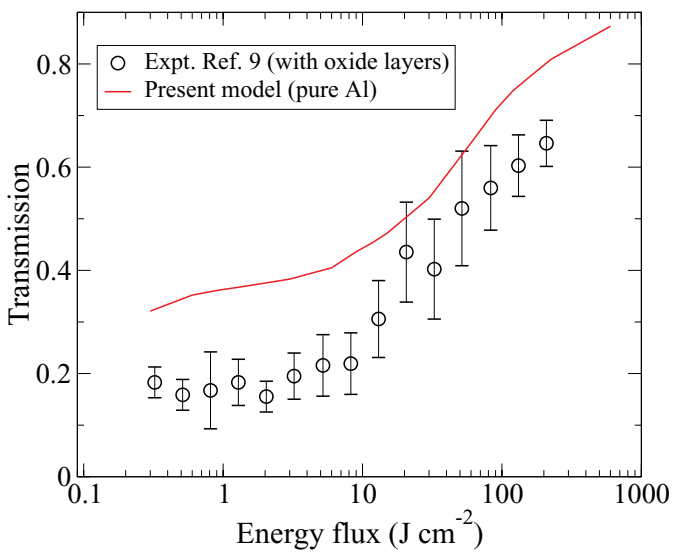

FIG. 10. (Color online) Transmission of an aluminum foil as a function of fluence. The solid line is the present calculation (see text). Experimental data points with error bars comes from Ref. [9]. It is worth noting that calculations do not take into account existing oxide layers.

\section{B. XUV- (92-eV) induced transparency of a thin Al target}

An experiment performed on FLASH has reported the demonstration of saturable absorption of $\mathrm{Al}$ in the soft $\mathrm{x}$ ray regime [9] by the creation of a highly uniform warm dense medium. The main result of that experiment is a clear curve giving the transmission of a XUV pulse through a 53-nm-thick Al target as a function of the pulse fluence. We found interesting to test the present model against these experimental data. For each fluence (in the range [0.1-1000] $\mathrm{J} \mathrm{cm}^{-2}$ ) and, given a pulse duration of $15 \mathrm{fs}$, we calculated a time-dependent transmission through the foil during the pulse. Our calculations used 20 cells and the same atomic model as used in the simulations presented above (Sec. IV A). The final result is a time-integrated transmission obtained by averaging instantaneous transmissions (calculated during the pulse) with a weight corresponding to the time-dependent XFEL intensity.

Results are plotted in Fig. 10 along with the experimental points of Ref. [9]. As in the experiment, the simulations exhibit a clear saturation behavior. However, one can see that the calculated transmission is shifted up compared with the experimental points. Here, one must be aware that our calculations do not take into account the effect of oxide layers $\left(\mathrm{Al}_{2} \mathrm{O}_{3}\right)$. Indeed, the existence of 10-nm layers of oxide on both side of the foil is explicitly mentioned in Ref. [9] and the CXRO value of the transmission of two (cold) 10-nm-thick $\mathrm{Al}_{2} \mathrm{O}_{3}$ layers is $T=0.48$ [39]. This factor can be applied to the low fluence part of the calculated saturation curve. For the intermediate and high fluence points where the XUV pulse starts to heat the sample, the effect of the oxide layers becomes less clear, and one can invoke a more or less important desorption of these layers. Keeping in mind these remarks, we shall notice a reasonable agreement between present simulations and the experimental results.

\section{X-ray (keV range) irradiation of a thick target}

In this section, we will present the simulation of a 1$\mu \mathrm{m}$-thick aluminum foil irradiated by a XFEL pulse of 80-fs (FWHM) duration, 1830-eV photon energy, $1.1 \times$
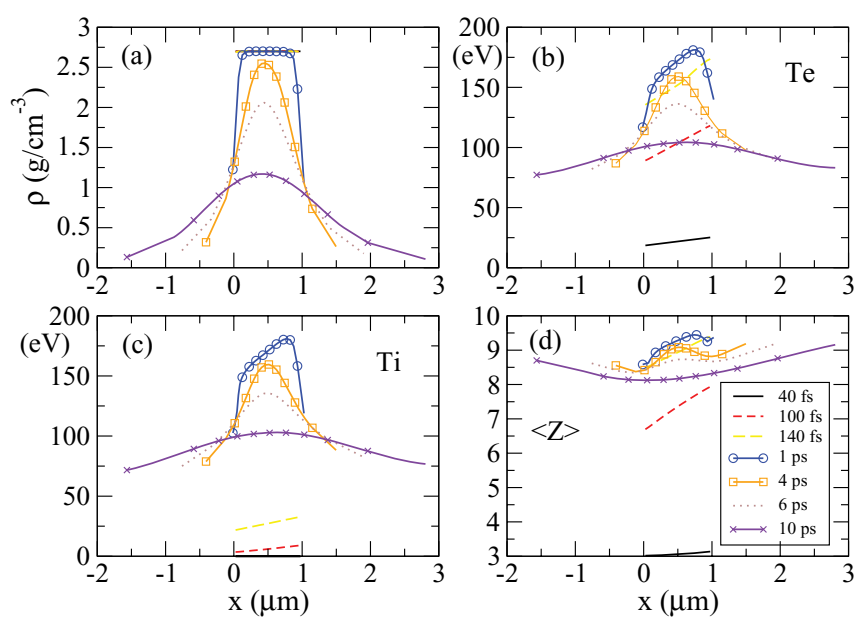

FIG. 11. (Color online) Snapshots of the $\rho, T_{e}, T_{i}$, and $\langle Z\rangle$ profiles at various times. The target is a $1-\mu \mathrm{m}$-thick foil. The XFEL $(1.1 \times$ $10^{17} \mathrm{~W} / \mathrm{cm}^{2}$ intensity, 80-fs FWHM, 1830-eV photon energy) comes from the right.

$10^{17} \mathrm{~W} / \mathrm{cm}^{2}$ peak intensity. Conditions of this simulation are close to the conditions of an experiment performed at LCLS [31]. Here, our intention is neither to discuss nor to interpret this specific experiment but rather to illustrate the capabilities of our model in the intense, $\mathrm{keV}$ regime where a strongly nonequilibrium atomic physics is expected.

In order to take into account the occurrence of one- and two-electron $K$-shell photoionization processes as well as a proper description of the whole population kinetics, our atomic model includes here 2792 configurations. Profiles of $\rho, T_{e}, T_{i}$, and $\langle Z\rangle$ at some selected instants are displayed in Figs. 11(a), 11(b), 11(c), and 11(d), respectively. For this photon energy and this thickness, the transmission is of the order of $50 \%$ so the profiles are rather flat while the target expands on both sides. As mentioned previously, the present model has also the capability of calculating synthetic spectra using the UTA formalism. Figure 12 displays x-ray spectra emitted by the created plasma at some selected times. Here again, they correspond to the emerging (from cell to cell) spectral emission along a line of sight normal to the target.

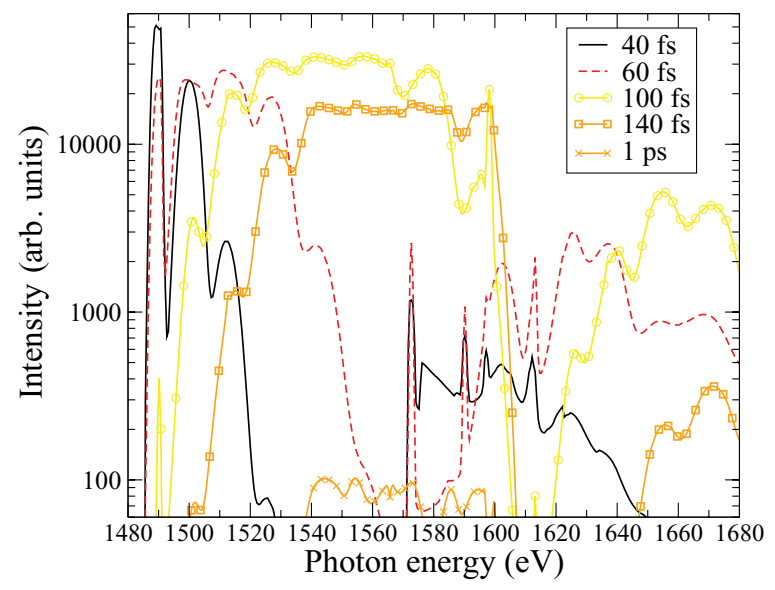

FIG. 12. (Color online) X-ray spectra of the radiated energy at some instants. XFEL conditions as in Fig. 11. 
As in Ref. [31], one can see here various emission peaks corresponding to the $K_{\alpha}$ in the several existing charge states. Also, it is worth noting that the early time emission taking place mostly during the XFEL pulse remains very specific compared with the later time emissions, which means that even time-integrated spectra can provide information on the solid density phase of the plasma. Also this strong $\mathrm{x}$-ray emission is much shorter than $1 \mathrm{ps}$ as the intensity at $t=1 \mathrm{ps}$ has dropped by a strong factor (two orders of magnitude) compared with the earlier times.

\section{SUMMARY AND CONCLUSIONS}

We have described a computer model to simulate the main physical processes occurring in a material under strong XFEL irradiation. Besides traditional aspects related to the hydrodyamical description of the laser-matter interaction, one needs a realistic atomic physics model describing the population kinetics of the many inner-shell ionized species. We have seen that, in addition to the photoionization and autoionization processes, collisional processes could play an important role. Another important quantity is the electron collisional frequency which must include the transition from a dense cold regime to a dense hot regime. To some extent, validity of the model has been set by calculating transmission and spectral emission and by comparing them to available experimental results. We noticed that, for the laser pulse durations considered here, the amount of heated matter is mainly controlled by the x-ray absorption depth and not by the depth of the thermal heat wave.

Here, the model has been applied to aluminum but, in its present state, it could be used for any metal or single-element material provided that an adequate choice of some adjustable parameters (involved in the electron collision frequency) is made prior to performing a simulation. In some cases where the $\mathrm{x}$-ray fluence is low and, thus, where the temperature remains low, it can be more judicious to impose a constant value for the electron-ion relaxation time during the entire simulation [40].

It would be interesting to determine at which time the present description becomes appropiate. A main approximation here is the supposed instantaneous thermalization of the free electrons (an approximation inherent to this kind of approach). We estimate that below a few tens of fs, the notion of electron temperature is probably uncertain.

To conclude, such an approach could be used as a predictive tool for the design or the interpretation of XFEL-matter interaction experiments as long as bulk properties of matter are sought in these conditions.

\section{ACKNOWLEDGMENTS}

The author wishes to acknowledge useful discussions with F. Rosmej.
[1] W. Akermann et al., Nat. Photon. 1, 336 (2007).

[2] P. Emma et al., Nat. Photon. 4, 641 (2010).

[3] D. Pile, Nat. Photon. 5, 456 (2011).

[4] LCLS homepage [http://lcls.slac.stanford.edu/].

[5] R. W. Lee et al., J. Opt. Am. B 20, 770 (2003).

[6] U. Zastrau et al., Phys. Rev. E 78, 066406 (2008).

[7] E. Galtier et al., Phys. Rev. Lett. 106, 164801 (2011).

[8] T. Dzelzainis et al., High Energy Density Phys. 6, 109 (2010).

[9] B. Nagler et al., Nat. Phys. 5, 693 (2009).

[10] N. Medvedev, U. Zastrau, E. Forster, D. O. Gericke, and B. Rethfeld, Phys. Rev. Lett. 107, 165003 (2011).

[11] S. P. Hau-Riege, R. A. London, H. N. Chapman, and M. Bergh, Phys. Rev. E 76, 046403 (2007).

[12] M. Fajardo, P. Zeitoun, and J.-C. Gauthier, Eur. Phys. J. D 29 69 (2004)

[13] S. Hau-Riege, AIP Conf. Proc. 1161, 100 (2009).

[14] D. S. Whittaker, E. Wagenaars, and G. J. Tallents, Phys. Plasmas 18, 013105 (2011).

[15] L. Spitzer, Physics of Fully Ionized Gases (Interscience, New York, 1956).

[16] T. W. Johnston and J. M. Dawson, Phys. Fluids 14, 722 (1973).

[17] M. I. Kaganov, I. M. Lifchitz, and L. V. Tanatarov, Zh. Eskp. Teor. Fiz. 31, 232 (1956) [Sov. Phys. JETP 4, 173 (1957)].

[18] Z. Lin, L. V. Zhigilei, and V. Celli, Phys. Rev. B 77, 075133 (2008).

[19] K. Eidmann, J. Meyer-ter-Vehn, T. Schlegel, and S. Huller, Phys. Rev. E 62, 1202 (2000).
[20] B. Chimier, V. T. Tikhonchuk, and L. Hallo, Phys. Rev. B 75, 195124 (2007).

[21] O. Peyrusse, Nucl. Fusion 44, S202 (2004).

[22] H. A. Scott and S. B. Hansen, High Energy Density Phys. 6, 39 (2010).

[23] O. Peyrusse, J. Phys. B 32, 683 (1999).

[24] R. D. Cowan, The Theory of Atomic Structure and Spectra (University of California Press, Berkeley, CA, 1981).

[25] R. E. H. Clark and D. H. Sampson, J. Phys. B 17, 3311 (1984).

[26] L. B. Golden and D. H. Sampson, J. Phys. B 13, 2645 (1980).

[27] D. L. Moores, L. B. Golden, and D. H. Sampson, J. Phys. B 13, 385 (1980).

[28] J. Bauche, C. Bauche-Arnoult, and O. Peyrusse, High Energy Density Phys. 5, 51 (2009).

[29] J. Bauche, C. Bauche-Arnoult, and M. Klapisch, Adv. Atom. Mol. Phys. 23, 131 (1987).

[30] C. A. Iglesias and R. W. Lee, J. Spectrosc. Radiat. Transfer 58, 637 (1997).

[31] S. M. Vinko et al. Nature (London) 842, 59 (2012).

[32] J. C. Stewart and K. D. Pyatt, Astrophys. J. 144, 1203 (1966).

[33] R. M. More (1981), LLNL technical report, No. UCRL-84991 (unpublished).

[34] G. B. Zimmerman and R. M. More, J. Spectrosc. Radiat. Transfer 23, 517 (1980).

[35] R. M. More, K. H. Warren, D. A. Young, and G. B. Zimmerman, Phys. Fluids 31, 3059 (1988). 
[36] Chandrani Bhattacharya and M. K. Srivastava, J. Appl. Phys. 102, 064915 (2007).

[37] R. D. Ritchmeyer and K. W. Morton, Difference Methods for Initial-Value Problems, 2nd ed. (Krieger, Melbourne, 1994).
[38] E. J. Caramana, M. J. Shashkov, and P. P. Whalen, J. Comput. Phys. 144, 70 (1998).

[39] CXRO [http://www.cxro.lbl.gov/].

[40] J. Gaudin et al., Phys. Rev. B 86, 024103 (2012). 